Emilio Bouza ${ }^{1,23,4}$

Benito Almirante 5,6

Julio García Rodríguez ${ }^{7}$

José Garnacho-Montero ${ }^{8}$

Miguel Salavert ${ }^{9}$

Patricia Muñoz $1,2,3,4$

Maurizio Sanguinetti10

\section{Biomarkers of fungal infection: Expert opinion on the current situation}

${ }^{1}$ Microbiología Clínica-Enfermedades Infecciosas. Hospital General Universitario Gregorio Marañón. Madrid. Spain

${ }^{2}$ Instituto de Investigación Sanitaria del Hospital Gregorio Marañón, Madrid, Spain.

${ }^{3}$ CIBER Enfermedades Respiratorias-CIBERES (CB06/06/0058).

${ }^{4}$ Departamento de Medicina. Facultad de Medicina. Universidad Complutense de Madrid. Spain.

${ }^{5}$ Servicio de Enfermedades Infecciosas. Hospital Universitari Vall d'Hebron. Barcelona. Spain.

${ }^{6}$ REIPI (RD16/0016/0003)

${ }^{7}$ Servicio de Microbiología. Hospital La Paz. Madrid. Spain

${ }^{8}$ Servicio de Unidad Clínica de Cuidados Intensivos. Hospital Virgen Macarena. Sevilla. Spain

${ }^{9}$ Servicio de Enfermedades Infecciosas. Hospital La Fe. Valencia. Spain.

${ }^{10}$ Chief Microbiology Department, Policlínico Gemelli, Catholic University. Rome. Italy.

Article history

Received: 14 October 2019; Accepted: 30 October 2019

\begin{abstract}
The introduction of non-culture-based diagnostic techniques is revolutionizing the world of microbiological diagnosis and infection assessment. Fungi are no exception, and the introduction of biomarkers has opened up enormous expectations for better management of these entities. Biomarkers are diverse, their targets are also diverse and their evaluation has been done preferably in an individualized use and with deficient designs. Less is known about the value of the combined use of biomarkers and the impact of the negativity of two or more biomarkers on antifungal treatment decisions has been poorly studied. Given the paucity of prospective, randomized and definitive studies, we have convened experts from different fields, with an interest in invasive fungal infections, to answer some questions about the current relevant use of fungal biomarkers. This document summarizes the answers of these experts to the different questions.
\end{abstract}

Key words: Invasive fungal infections, Intensive Care, Critical Care, Candidemia, Invasive candidiasis, invasive aspergillosis, fungal biomarkers, CAGTA, 1-3B-D-Glucan, Mannan, Anti-Mannan, T2Candida, Galactomannan, antifungal stewardship

Correspondence:

Emilio Bouza MD, PhD

Instituto de Investigación Sanitaria Gregorio Marañón

Servicio de Microbiologia Clinica y E. Infecciosas

C/ Dr. Esquerdo, 46

28007 Madrid, Spain

Phone: +34- 91- 58684 53/Fax: +34- 91- 5044906

E-mail:emilio.bouza@gmail.com

Alternative corresponding author

Patricia Muñoz, MD, PhD

Servicio de Microbiologia Clinica y Enfermedades Infecciosas

Hospital General Universitario Gregorio Marañón

C/ Dr. Esquerdo, 46

28007 Madrid, Spain

Phone: +34- 91- 58684 52/Fax:+34- 91- 5044906

E-mail:pmunoz@micro.hggm.es

\section{Biomarcadores de infección fúngica: Opinión de expertos sobre la situación actual}

\section{RESUMEN}

La introducción de técnicas de diagnóstico no basadas en cultivo está revolucionando el mundo del diagnóstico microbiológico y de la aproximación a las infecciones. Los hongos no son una excepción, y la introducción de biomarcadores ha abierto enormes expectativas para una mejor manejo de estas enfermedades. Hay diversos biomarcadores cuyo significado es también diverso pero su evaluación se ha hecho preferentemente en un uso individual y con estudios con distitnos diseños. Se sabe menos sobre el valor de la combinación de biomarcadores y el impacto de la negatividad de dos o más de los mismos en las decisiones de tratamiento antifúngico ha sido poco estudiado. Dada la escasez de datos prospectivos, en estudios aleatorizados y definitivos, hemos convocado a expertos de diferentes campos con un interés en las infecciones micóticas invasivas, para responder a algunas preguntas sobre el uso actual y relevante de los biomarcadores fúngicos. Este documento resume las respuestas del grupo de expertos a las preguntas que se les formularon sobre el tema.

Palabras clave: Infección fúngica invasora, Cuidados intensivos, Cuidados críticos, Candidemia, Candidiasis invasora, Aspergilosis invasora, biomarcadores fúngicos, CAGTA, 1-3ß-D-Glucano, Manano, Anti-Manano, T2Candida, Galactomanano, política de antifúngicos

\section{INTRODUCTION}

Invasive Fungal Infection (IFI) is becoming increasingly important due to a series of circumstances, including the growth of a population with multiple risk factors and immunosuppressed, in which the control of bacterial infections is more effective. Exposure to both endogenous and exogenous fungi is favored by hospitalization and procedures that injure cutaneous-mucous barriers. Finally, the availability of effective 
antifungal agents determines the need for rapid and accurate diagnosis.

Culture-based diagnostic media have a recognized and clear role in the diagnosis of invasive mycosis but their deficiencies are also known, particularly in sensitivity and also in speed.

Therefore, it is essential to implement the diagnosis of invasive mycosis with non-culture-based techniques among which biomarkers are already an essential part. However, the position of biomarkers of IFls in everyday clinical use is far from uniform in all hospitals, the performance of some techniques is under discussion, their interpretation is not always simple and the existing bibliography is sometimes biased for many reasons.

This has led to the convening of a series of experts from the fields of Microbiology, Infectious Diseases and Intensive Care, confronting them with a series of questions that seemed relevant. The open-door meeting took place in Madrid on 23 May 2019 on the occasion of the National Congress of the Spanish Society of Infectious Diseases and Clinical Microbiology (SEIMC). This document tries to reflect the main issues discussed, the documentation provided on them and the conclusions that were agreed by the group.

The final document, after having been edited and revised, has been approved by all the participants and represents the opinion of all of them and not necessarily of the institutions to which they belong.

\section{MATERIAL AND METHODS}

The questions were chosen by the coordinators and accepted by all the speakers. The document, edited in a first draft has been sent to all co-authors for their corrections and amendments. The final document has been reviewed and approved by all authors.

Next, we will review the questions posed, the arguments provided and the conclusions reached in each of them.

QUESTION 1. Could the importance of invasive fungal infection (IFI) in Spain be quantified? What proportion of it is confirmed by culture?

\section{Dr. Benito Almirante}

\section{Background:}

Rodriguez-Tudela et al attempted to estimate the incidence of IFIs in Spain in a 2015 publication [1]. They estimated that approximately 9.52 episodes of invasive candidiasis (IC) occur per 100,000 inhabitants and that in the case of Invasive Aspergillosis (IA) the figure was 2.75 episodes/100,000 inhabitants. The number of cases of mucormycosis was significantly lower and was estimated at 0.04 episodes per 100,000 inhabitants per year. They also estimated the figures for certain groups of patients and they reported that $4 \%$ of all solid organ transplants and $1.6 \%$ of all patients with malignant hematological diseases had, at one time or another, an IFI.

Table 1 lists some studies particularly aimed at evaluating the incidence of candidemia in Spain. As can be seen in table 1, the figures per 100,000 inhabitants per year range from 4.3 to 8.1 episodes and the data estimated with the denominator of 1.000 hospital admissions vary between 0.53 and 1.09 episodes.

At the time of detection of the episode of candidemia, patients in Spain were mostly hospitalized (88\%) and a third (35\%) were admitted to Intensive Care Units (ICUs). The medical and surgical areas represented $28 \%$ and $21 \%$, respective$l y$, and the pediatric areas $3 \%$. A miscellany from other places represented only 1\% [2].

The three invasive entities caused by Candida were candidemia in the absence of organ candidiasis, candidemia associated with organ disease and candidiasis of organs without accompanying candidemia. The difficulties of diagnosis by conventional methods $[7,8]$ arise because the sensitivity of blood cultures does not exceed 50\% and, when available, is often delayed more than 48 hours. In the case of organ IC, there are only positive reliable cultures in approximately $50 \%$. Invasive procedures are frequently required to obtain proper samples but are rarely possible.

Regarding the conventional diagnosis of IA $[9,10]$ the challenges are not minor. Signs and symptoms are often nonspecific, it is difficult to distinguish colonization from infection, blood cultures are practically always negative and it is also difficult or impossible to perform invasive techniques for obtaining proper samples. The use of non-culture-based biomarkers is therefore indispensable.

\begin{tabular}{l|lcccc}
\hline Table 1 & \multicolumn{1}{l}{ Studies on the incidence of candidemia in Spain } \\
\\
\multicolumn{1}{l}{ Reference } & Period & N cases & $\times 10^{5}$ inhabitants & $\times 10^{4}$ days of hospital stay & $\times 10^{3}$ hospital admissions \\
\hline Almirante B, 2005 [3] & $2002-03$ & 345 & 4.3 & 0.73 & 0.53 \\
Rodríguez-Hernández MJ, 2011 [4] & $2005-06$ & 220 & ND & 0.8 & 0.58 \\
Cisterna R, 2010 [5] & $2008-09$ & 984 & ND & ND & 1.09 \\
Pemán J, 2012 [6] & $2009-10$ & 1,357 & ND & ND & 0.92 \\
Puig-Asensio M. CANDIPOP Project, 2013 [2] & $2010-11$ & 773 & 8.1 & 1.36 & 0.89 \\
\hline
\end{tabular}




\section{Conclusion:}

Invasive infection caused by Candida is estimated in Spain at 9.52 episodes per 100,000 inhabitants/year and that caused by Aspergillus at 2.75 episodes. Confirmation with culture occurs in less than $50 \%$ of cases due to the frequent negativity of blood cultures and the difficulty of obtaining deep samples of ordinarily sterile tissues or fluids.

QUESTION 2. How should "biomarker of fungal infection" be defined? What are the most important ones at this time?

\section{Dr. Benito Almirante}

\section{Background:}

A biomarker of fungal infection is a "Biological product from the structure of the fungus/yeast, capable of being detected by "unconventional" techniques, allowing (sometimes) an early and/or anticipated diagnosis of an invasive fungal infection" [11].

Most common ones are summarized in table 2 and are, in the case of Candida, mannan and anti-mannan, antibodies against Candida germinal tubes (CAGTA), 1-3- $\beta$-D-glucan (1-3-BDG), nucleic acids and the T2Candida nanodiagnostic panel. In the case of IA, the most commonly used are: galactomannan (GLM) in serum, BAL or other samples, 1-3- $\beta D G$, nucleic acids (serum, blood or other samples) and Aspergillus lateral flow assay (A-LFD) technology [9, 11-13].

\section{Conclusion:}

A biomarker of Invasive Fungal Infection is a biological product from the structure of the fungus/yeast that can be detected by non-culture-based techniques. At this time, the most commonly used in the case of Candida are mannan and anti-mannan, antibodies against Candida germinal tubes (CAGTA), the 1-3- $\beta-D-$ glucan, the detection of nucleic acids, and the T2Candida nanodiagnostic panel. In the case of Invasive Aspergillosis, galactomannan, 1-3- $\beta$-D-glucan, nucleic acids, and Aspergillus lateral flow assay (A-LFD) technology are the more common.

QUESTION 3. What is CAGTA and what does it contribute to the diagnosis of invasive candidiasis?

\section{Dr. Julio García Rodríguez}

Background:

The anti-micelium antibody detection system for the diagnosis of invasive candidiasis (IC) (CAGTA) is a technique developed at the University of the Basque Country (Spain) and marketed in the form of indirect immunofluorescence [14]. It was originally designed for the detection of antibodies against antigens expressed in the mycelial phase of Candida albicans when it is invading tissues. However, it was soon found to be useful for the diagnosis of infections by other Candida species. It is a technique

\begin{tabular}{l|l}
\hline Table 2 & $\begin{array}{l}\text { Biomarkers of invasive fungal infection } \\
\text { of common use }\end{array}$ \\
\hline Biomarkers of invasive candidiasis & Biomarkers of invasive aspergillosis \\
\hline Mannan-Anti-mannan & Galactomannan (Blood, BAL) \\
CAGTA & Aspergillus Lateral Flow Assay \\
1-3-B-D- Glucan & 1-3-B-D- Glucan \\
Nucleic acids & Nucleic acids \\
Nanodiagnostic T2Candida & \\
\hline
\end{tabular}

CAGTA: Candida albicans germ tube antibody

that allows to quantify these antibodies and it is commercialized for the diagnosis of IC.

The CAGTA test, in individualized use, has been evaluated on numerous occasions with different results. In a recent meta-analysis, the authors found an overall sensitivity of $66 \%$ with a specificity of $76 \%$ [15]. In addition, in some studies, it has been possible to relate a higher antibody titer with a better prognosis in patients admitted to the ICU, so that it could be used as a prognostic marker depending on its kinetics [16].

Due to its limited diagnostic value, it has been attempted to be used in combination with other biomarkers such as 1,3-BDG, or antibodies and/or mannan antigens. Recently it has also been combined with the T2Candida magnetic resonance system. According to the different studies, the main usefulness of the combination of these biomarkers lies in their high negative predictive value. This implies that when an antifungal treatment is empirically established, the negativity of two of these markers could be sufficient to safely withdraw the treatment [17-19].

\section{Conclusion:}

CAGTA is a Candida antimicelial antibody detection system, developed in Spain and commercialized in the form of indirect immunofluorescence. Its negativity, when it coincides with that of other biomarkers, may allow the suspension of antifungal treatments initiated on an empirical basis.

QUESTION 4. What are the indications and limitations of the use of Galactomannan (GLM) in a general hospital at the present time?

\section{Dr. Julio García Rodríguez}

\section{Background:}

After the study published by the group of Duarte et al. [20] in haematological patients with high risk of fungal infection, who received prophylaxis with posaconazole, and later corroborated in patients on prophylactic treatment with micafungin [21], it has been determined the poor role that the detection of GLM alone plays in these populations when used 
as a weekly screening for the initiation of early preemptive antifungal therapy. Both groups of researchers have pointed out that in a setting where the prevalence of IA is reduced to less than $2 \%$, the pre-test probability of GLM falls dramatically, as does the positive predictive value. Therefore, any positive in this situation will be more likely to be a false positive than a true value. These false results may lead these patients to other more or less annoying confirmatory diagnostic tests and, on many occasions, to the initiation of nonrequired antifungal treatments. In short, an increase in the risk of iatrogeny and expenditure.

Although these studies currently advise against the use of GLM as a diagnostic anticipation tool in hematological patients undergoing antifungal prophylaxis, the test is still very useful in those situations in which the patient already has a clear clinical suspicion of IA [22]. There are also other circumstances in which this test is very useful, which are summarized below:

Detection in bronchoalveolar lavage (BAL). The latest reviews and meta-analyses have confirmed that detection of GLM in BAL is more sensitive than in serum, both in hematological and non-hematological patients $[23,24]$.

Extrapulmonary IA. Other situations in which the determination of GLM may be useful is in extrapulmonary forms of IA such as the case of cerebrospinal fluid for cerebral localizations [25] or peritoneal fluid for the diagnosis of peritonitis [26].

Other applications. Recently, GLM has also been considered useful in the follow-up of high-risk patients in hospitals with undergoing works [27], as a prognostic marker of chronic aspergillosis and as a diagnostic test for disseminated fusariosis [27].

Difficulties. However, the format currently marketed by a microtiter plate capture ELISA makes it very difficult to use the test with only one or a few samples. Initially, a latex agglutination for the detection of GLM antigen existed in a commercialized form, which was substituted by the capture ELISA due to the greater analytical sensitivity of the latter. The ELISA method is useful when studying a large number of patients at risk of IA in a systematic way. New formats are now needed to facilitate its use with a single sample. These new systems must be able to quantify the fungal load and be easy to carry out. One model would be the new immunochromatography based on "Lateral Flow", which is a very simple, but difficult to quantify, technique. The new 1-3-BDG detection test using WAKO turbidimetry could also be a suitable format. Finally, the new monotest using chemiluminescence developed by Vircell, in the absence of a clinical evaluation and published studies, could respond to these new diagnostic needs.

\section{Conclusion:}

Galactomannan is a useful diagnostic test for the investigation of Invasive Aspergillosis in patients with clinically suggestive conditions. It can be performed not only in blood but also in CSF, bronchoalveolar lavage or peritoneal fluid. Its systematic and periodic use is not recom- mended in non-symptomatic hematological patients who are receiving antifungal prophylaxis.

QUESTION 5. To whom should a 1-3 $\beta$-D-Glucan level be determined?

\section{Dr. Miguel Salavert}

\section{Background:}

The performance of classical diagnostic tests on patients who are to be, or are undergoing, antifungal treatment is usually limited to patients who fall within the definition of pre-emptive treatment [28]. However, the use of biomarkers can also be useful to identify therapeutic or prophylaxis failures and can help to confirm or rule out disease in patients on empirical or early treatment or help to decide the duration and prognosis of patients with confirmed diagnosis.

The $1-3-\beta D G$ is a component of the fungal wall composed mainly of glucose polymers joined by 1-3 glucosidic bonds that form the skeleton of the fungal wall. As the fungus grows, part of this compound is released into the blood, where it can be measured. Beta-glucans are able to activate the blood coagulation cascade of Limulus polyphemus, the North American horseshoe crab [29], which forms the basis of the diagnostic test. It is produced by fungi such as Aspergillus sp, Candida sp, Pneumocystis sp. Coccidioides sp, and Histoplasma sp. but is not produced in Cryptococcus sp and Mucorales.

The positivity of 1-3-BDG can anticipate by an average of 10 days diagnostic clinical manifestations of invasive fungal infections [30]. In a study conducted by Tissot et al. [31], in patients with intra-abdominal candidiasis without candidemia, 1-3-BDG became positive an average of 5 days before confirmation of the diagnosis. In addition, severe sepsis and mortality in this study were higher in patients with higher levels and persisted high, rather than falling, in patients with poor progression.

Determination of 1-3-BDG can produce both false positives and false negatives. Causes of false positivity include hemodialysis with cellulose membranes, exposure of tissues to cotton gauze and other materials that may contain glucans, administration of blood products through filters, and some antimicrobials such as amoxicillin-clavulanic, piperacillin-tazobactam, co-trimoxazole, colistin, cefepime, ertapenem, and others that suggest contamination with fungal fragments during production [32]. In general, in these cases, the positivity of the test disappears quickly after suspension of the antibiotic or dilution of the sample. There are also false positives described in patients with bacteremia, particularly gram-negative bacteremia, but some studies show that a substantial proportion of bacteremic and 1-3-BDG-positive patients have simultaneously possible, probable or proven mycoses [33]. Therefore, false positives may occur in patients with bacteremia but this is rare, and the presence of an underlying fungal infection or causes of false positivity other than bacteremia should always be suspected. Although Candida colonization does not cause false positives, patients with mucositis may have higher levels of 1-3-BDG [34].

The classical (Fungitell) determination technique (using kinetic ELISA) has important practical limitations in an assistance labora- 
tory as it almost never allows "real time" determination due to the need of batching serums for joint processing. It is also a long (approx. 3 hours) and laborious technique to perform (not suitable for inexperts). Only $10 \mu \mathrm{l}$ of sample (serum) are used, and the minimum pipetting error can generate important errors in the result (usually false negatives). In the new format of the technique (WAKO/FUJIFILM), based on the detection of 1-3-BDG by chemiluminescence in MONOTEST format, it allows to analyze sera individually, in much less time (45-90 minutes) and uses more sample volume (70 $\mu \mathrm{l})$.

Today, there is no doubt that the addition of 1-3-BDG to a hospital where immunocompromised patients are cared is a necessity, which is reinforced by the incorporation of the new test presentations, easier to do and more functional. The determination of $1-3-B D G$ is already included in the most recent diagnostic guidelines for invasive mycoses $[35,36]$, although the frequency of the determinations is not clearly specified. Frequently, the rhythm has to do with the laboratory's possibilities to perform the test, which is often done in batches, when enough samples have been accumulated to make the test profitable. This causes clinicians to receive the test at an inadequate pace that does not anticipate clinical events and is inefficient. The availability of a test that allows daily performance, without wasted materials and human resources, can change the clinical use of the test and its diagnostic and prognostic effectiveness.

Today, 1-3- $\beta D G$ can be a powerful tool in an institution as long as it is properly used and interpreted.

\section{Conclusion:}

The presence of 1-3- $\beta-D$ Glucan should be determined in patients with suspected invasive fungal disease, or as a follow-up to confirmed mycoses, provided that the timing of the determinations, data return, and test interpretation are properly applied.

QUESTION 6. What is the value of a positive or negative 1-3- $\beta-D$ Glucan? What is the value of its evolution?

\section{Dr. Miguel Salavert}

Background:

A positive test for 1-3-BDG, if the causes of false positives mentioned above are excluded, may suggest the presence of an IFI, not yet demonstrated by other methods, discriminates between colonization and infection in obviously colonized patients, reinforces the results of other diagnostic tests and contributes to the performance of predictive scores.

In the meta-analysis of Karageorgopoulos et al. [37] the results of the $1-3-\beta D G$ of patients with or without criteria of certainty or probability of IFI, following the criteria of the EORTC, are compared in the analyzed studies, excluding cases of $P$. jirovecii infection. Of the 594 patients who met this condition, in the 16 studies analyzed, the overall sensitivity of the test was $76.8 \%$ and its specificity $85.3 \%$. AUCROC was 0.89 and it is concluded that $1-3-B D G$ is a test with diagnostic precision to differentiate patients with IFI proven or probable from those who do not have it.

Lamoth's et al. meta-analysis focuses on hematological pa- tients [38] and includes 6 cohort studies totaling 1,771 adults of whom 414 had IFls (215 tested or probable). They did not detect discrepancies between the various commercially available tests. When the existence of two consecutive positive tests is used as a positive value, the sensitivity and specificity were respectively $50 \%$ and $99 \%$. For an estimated prevalence of $10 \%$ of IFls the PPV and NPV were respectively 83.5\% and 95\%.

In a meta-analysis based exclusively on prospective studies [39], a total of 1,068 patients from 11 studies were examined and the cumulative data obtained were the following, with 95\% confidence intervals: Sensitivity 75\%, Specificity $87 \%$, Positive likelihood ratio 5.85, Negative likelihood ratio 0.30 , diagnostic odds ratio 19.53 , and area under the summary receiver operating characteristic curve, of 0.89 . This suggests that this is a clinically useful test with a high ability to distinguish between patients with and without IFI that should be used together with other clinical and microbiological data.

The interpretation requires always to exclude the most frequent causes of false positive summarized in table 3 and false negatives summarized in table 4 .

In the case of P. jirovecii pneumonia (PJP) [40] 14 studies allowed to analyze the result of 1-3-BDG in 357 cases and 1,723 controls. Cumulative data showed 95\% sensitivity and $86 \%$ specificity. The positive and negative likelihood ratios were 6.9 and 0.06 , respectively. The area under the HSROC curve was 0.965. The serum 1-3-BDG determination showed excellent sensitivity and very good specificity in the diagnosis of PJP.

Nucci et al [41] have recently provided interesting data on the use of 1-3-BDG in the diagnosis and prognosis

\section{\begin{tabular}{l|l} 
Table 3 & Some causes of false-positive results in
\end{tabular} the 1-3 ßDG test}

- Contamination of laboratory material with glucans.

- Bacteremia due to Streptococcus spp. or some Gram-negative bacilli such as Pseudomonas spp.

- Contact with surgical sponges and gauzes.

- Hemodialysis patients with cellulose containing filters.

- IV treatment with immunoglobulins, albumin or coagulation factors.

- Antibacterial IV treatment with antibiotics such as amoxicillin-clavulanic or piperacillin-tazobactam.

- Antineoplastic treatments with Lentinane or Polysaccharide k.

Table 4 Some causes of false-negative results in the 1-3-ßDG test

- Hyperpigmented serums (bilirubin, triglycerides).

- Antifungal treatment (prophylaxis, empirical).

- Azithromycin or pentamidine IV. 
of disseminated fusariosis. In a group of 13 cases of fusariosis, 12 had at least one positive test and in 11 of them the test was positive before diagnostic confirmation by other methods. Once treatment was started, the evolution of the determination was also interesting. The 1-3-BDG continued to grow in patients who died within 30 days, while it was maintained or decreased in those who survived more than 1 month.

The determination of $1-3-\beta D G$ has also been carried out on material from bronchoalveolar lavages (BAL). In a meta-analysis that included data from 6 studies and 838 patients (138 with proven or probable IFI), the accumulated sensitivity showed marginal efficacy data, that do not allow interpreting these results in isolation $[42,43]$.

Another aspect to discuss is the value of 1-3-BDG follow-up in patients already diagnosed with IFls. The 1-3BDG is a good biomarker of IFls follow-up if the results are brought quickly to the clinician, which may allow the possibility of antifungal change or de-escalation of them. The control of the evolution of the fungal load can also influence the surgical or instrumental decision making and even in some mycoses allow epidemiological follow-up. In this field, however, methodologically correct studies are needed before clear conclusions can be drawn. Pini et al [44] have analyzed the prognostic potential of 1-3-BDG in 253 patients with IFIs who conclude with a positive (177 episodes) or negative (76 episodes) evolution. Using an interpretive algorithm based on two different breakpoints of significance, they were able to predict evolution in $82 \%$ of cases.

\section{Conclusion:}

Two consecutive positive blood tests for 1-3-BDG, excluding the most frequent and obvious causes of false positivity, are associated with a high suspicion of invasive fungal infection. Its persistence or elevation in confirmed mycoses in antifungal treatment may be associated with poor prognosis.

QUESTION 7. What value does the simultaneous determination of several biomarkers have in daily clinical practice?

\section{Dr. Patricia Muñoz}

\section{Background:}

Biomarkers do not provide categorical diagnoses, but are Bayesian parameters, which assign a probability of infection (according to pre and post test probabilities). There are several reasons for using more than one biomarker. For example, when the suspected diagnosis is broad (Candida, filamentous fungi, etc.) and the possibilities of detection are to be expanded. With false negatives in mind, several biomarkers are used before suspending an empirically initiated antifungal treatment. On the contrary, and trying to avoid the false positive effect, combinations of biomarkers are used to avoid unnecessary treatments.
Martínez-Jiménez et al. used a combination of Candida biomarkers in a group of 100 patients with bacteremia and candidemia including CAGTA, Mannan/Antimannan (MN/AMN) and 1-3-BDG (with different cut-off points) in patients undergoing antifungal treatment. Biomarkers, used one by one, had limited sensitivity and specificity. Conversely, various combinations [45] such as the combination of CAGTA and 1-3-BDG had, globally, a NPV of $97 \%$ for the diagnosis of IC. The best behavior of this combination of tests was observed in ICU patients.

Pini et al. [46] retrospectively studied the presence of CAGTA and 1-3-BDG in stored serum samples from 29 patients with proven IC and 28 controls (9 with demonstrated bacteremia and 9 with negative blood cultures). The association of the two markers clearly increased the sensitivity and accuracy of the separate tests, with the two tests together achieving the following values: 97\% sensitivity, 84\% specificity, 78\% PPV, 95\% NPV, and 84\% accuracy.

Another possible combination that has been studied is the combination of 1-3-BDG and procalcitonin (PCT) [47], in patients admitted to intensive care. The study evaluates the significance of 1-3-BDG positive with PCT less than 2. The combination gives the following results: sensitivity of $96 \%$, specificity of $60 \%$, PPV of $65 \%$ and NPV of $95 \%$.

The combined use of 1-3-BDG with GLM as early markers of fungal infection was used in a monocentric study that enrolled 270 suspected episodes of IFI, 58 proven or probable IA, 27 proven IC, 11 possible IC, 16 P. jirovecii pneumonia (PJP) and 4 episodes of other IFI and 154 non-IFI controls. The combination of 1-3-BDG and GLM increased sensitivity from 60\% to $83 \%$ in hematological patients [48].

A recent meta-analysis included 13 studies evaluating combinations of GLM, 1-3-BDG, and aspergillus-lateral flow device (A-LFD) for the diagnosis of IA [49]. Authors included 1,513 patients. Pooled GLM and 1-3-BDG combination data showed sensitivity of $49 \%$, specificity of $98 \%$, Positive Likelihood Ratio- PLR 32 (95\%Cl 5.36-187.37), NLR 0.52 (95\%Cl 0.32-0.84) and DOR 61.23 (95\%Cl 6.96-538.90). The combinations clearly increased their diagnostic value.

\section{Conclusion:}

The simultaneous combination of two or more biomarkers improves both the sensitivity and the negative predictive value of the tests used separately and is therefore clinically useful.

QUESTION 8. Does the negativity of biomarker combinations allow early suspension of antifungal treatment?

\section{Dr. Patricia Muñoz}

\section{Background:}

Rouzé et al. [50] studied the impact of a strategy based on the use of biomarkers in the early discontinuation of empirical antifungal treatments in ICU. A total of 
110 patients were randomized to an antifungal suspension strategy based on the combined use of 1-3-BDG, mannan, and anti-mannan in serum on days 0 and +4 , compared to the usual standard, based on clinical practice guidelines and 14 days of treatment. In the biomarker-guided group, treatment was discontinued early in 29 of 54 patients compared with only 1 out of 55 early suspension in the group managed without biomarkers ( $54 \%$ vs $2 \%, p<$ $0.001)$. The median duration of antifungal treatment was 6 days versus 13 days, with no further differences in the occurrence of IC or differences in mortality between the two groups.

A cohort of 549 high-risk hematological patients undergoing antifungal prophylaxis were followed with antigenic determination and PCR [51]. The combination of techniques showed great utility in the management of IA, with high sensitivity (98\%) and NPV (100\%) when both tests were used together, allowing both early treatment and early suspension of antifungals. Biomarkers preceded clinical signs in $85 \%$ of cases of proven or probable IA.

The screening of IA is essential to decrease the empirical use of antifungals. A meta-analysis of high-risk patients combining GLM and PCR weekly determination, included 13 studies and 1,670 patients [52]. The study concluded that the negativity of all the tests makes it possible to obviate the need to administer antifungal agents with an NPV of $100 \%$, while the presence of at least 2 positive tests is highly suggestive of active infection with a PPV of $88 \%$.

\section{Conclusion:}

The negativity of two or more biomarkers of invasive fungal infection supports the suspension of many empirical treatments and is an essential element of an adequate antifungal use policy, although prospective and well-designed studies are still needed to reinforce this concept.

QUESTION 9. Can biomarkers allow, in the future, the individualization of the duration of antifungal treatment?

\section{Dr. José Garnacho-Montero}

\section{Background:}

In a study carried out in Spain, and already commented [53], 100 patients at high risk of IFI (63 in the ICU) were selected with empirical antifungal therapy. An IC could be tested in 30\% of them. Determinations of CAGTA and 1-3-BDG were made on days 0, 3 and 5 after starting empirical antifungal therapy. The NPV of the combination of both tests was $97 \%$ and among non IC patients, all biomarkers were negative in 31/58 patients (53\%) and there were 27 false positive results. False positives were more common in the ICU (51.2\%) than in general hospital wards (33.3\%).

Posteraro et al. [54], in Massimo Antonelli's group, determined the effect of a 1-3-BDG use strategy in patients at high risk of IC in an ICU. Out of a total of 198 patients, 63 were 1-3-BDG positive (cut-off value for BDG positiv- ity $>80 \mathrm{pg} / \mathrm{mL}$ ) at some point ( 47 with candidemia and 16 with probable IC) and all of them received antifungal treatment (31.8\%). In the 135 negative 1-3-BDG patients, $110(55.5 \%)$ never received antifungal treatment and 25 $(13 \%)$ received it only initially. Candidemia was only diagnosed in 1 patient who had not received previous antifungal treatment. With this approach, the authors reported that they were able to avoid unnecessary antifungal treatment in approximately $73 \%$ of potentially treatable patients and reduce duration in another $20 \%$ of cases.

The work of Rouze et al.[50] who prospectively study the impact of a strategy of rapid suspension of empirically initiated antifungal treatments based on the results of 1-3-BDG has also been commented on. This is a randomized study comparing a group in which treatment is done according to standard recommendations and another that relies on biomarker results. As already commented, the biomarker-based strategy was basically safe and allowed a very substantial reduction in the unnecessary use of antifungals.

The data have therefore so far focused on the use of biomarkers to suspend theoretically unnecessary treatments. In the case of patients with necessary treatments, we have not found data that would allow the evolution of biomarkers to be used as a strategy to shorten or adjust the duration of treatment either in candidemia without organ candidiasis, or in candidemia with candidiasis of organs, or in the case of candidiasis of organs without accompanying candidemia. The dissociation between time to the negativity of blood cultures and time to the negativity of some biomarkers (T2Candida) allows speculation on their future use $[55,56]$.

\section{Conclusion:}

There are no data that permit to state, at this time, that biomarkers and their evolution can be used to adjust the duration of antifungal treatment in patients with invasive fungal infection.

QUESTION 10. To what extent are biomarkers going to modify the attitude towards invasive candidiasis in Intensive Care Units?

\section{Dr. José Garnacho-Montero}

\section{Background:}

Invasive fungal infections (IFIs) increase their incidence in ICUs where approximately $80 \%$ are caused by Candida spp. and 0.3-19\% by Aspergillus spp.[57]. The problem of IC/candidemia in ICUs in Europe has been recently recalled with data from the EUCANDICU study which included 23 ICUs in 9 European countries. The cumulative incidence was 7 episodes per 1,000 admissions with great variability between centers. Crude mortality at 30 days was $42 \%$ [58]. We are, therefore, faced with a problem of enormous severity that requires intervention.

The problems related to IF Is in ICU patients are so complex that they need to be addressed by collaborative groups, as suggested in the recommendations of the European Society of 
Intensive Care Medicine (ESICM) and the Critically III Patients Study Group of the European Society of Clinical Microbiology and Infectious Diseases (ESCMID) [59] and should result in the establishment of a stewardship for the rational use of antifungals specifically focused on ICU patients.

Biomarkers are, and will be, an essential element of a policy of diagnostic anticipation and rational use of antifungals. The combined use of CAGTA and 1-3-BDG in two consecutive samples of ICU patients in Spain, showed $90 \%$ sensitivity, 42 $\%$ specificity and $97 \%$ NPV, also allowing a reasonable discriminatory use between colonization and invasion in patients with severe intra-abdominal diseases [17].

Candida biomarkers such as CAGTA, 1-3-BDG and others need even more research and studies to demonstrate the clinical impact of their systematic use. Future studies should specifically address the use of different diagnostic and therapeutic strategies in patients with intra-abdominal candidiasis [57].

Finally, it is clear that the availability of biomarkers will not delay empirical treatment and therefore will probably reduce mortality. Biomarkers will permit suspension of treatments if negative, in the relevant clinical context, and avoid the overuse of antifungals with considerable ecological and economic benefits.

\section{Conclusion:}

Candida biomarkers introduce a new era in Intensive Care Units. They will allow a diagnostic and therapeutic anticipation with the consequent decrease in mortality, an early discontinuation of unnecessary empirical treatments. Biomarkers will represent an excellent contribution to antifungal stewardship

\section{FINAL REMARK}

At the time of finishing the edition of this manuscript, during the month of October 2019, a Guideline of the American Thoracic Society has appeared, referring to the microbiological diagnosis of pulmonary fungal infections that we consider of particular relevance and we recommend to read to the readers of this text [60].

\section{ACKNOWLEDGEMENTS}

This symposium was held thanks to an unrestricted grant from the company Vircell (Spain).

\section{REFERENCES}

1. Rodriguez-Tudela JL, Alastruey-Izquierdo A, Gago S, Cuenca-Estrella $M$, Leon $C_{1}$ Miro JM, et al. Burden of serious fungal infections in Spain. Clin Microbiol Infect. 2015;21(2):183-9. DOI 10.1016/j. cmi.2014.07.013.

2. Puig-Asensio M, Padilla B, Garnacho-Montero J, Zaragoza 0 , Aguado JM, Zaragoza $R$, et al. Epidemiology and predictive factors for early and late mortality in Candida bloodstream infec- tions: a population-based surveillance in Spain. Clin Microbiol Infect.2014;20(4):0245-54. DOI 10.1111/1469-0691.12380.

3. Almirante B, Rodriguez D, Park BJ, Cuenca-Estrella M, Planes AM, Almela $M_{1}$ et al. Epidemiology and predictors of mortality in cases of Candida bloodstream infection: results from population-based surveillance, Barcelona, Spain, from 2002 to 2003. J Clin Microbiol. 2005;43(4):1829-35. DOI 10.1128/jcm.43.4.1829-1835.2005.

4. Rodriguez-Hernandez MJ, Ruiz-Perez de Pipaon M, Marquez-Solero M, Martin-Rico P, Caston-Osorio JJ, Guerrero-Sanchez FM, et al. Candidemias: multicentre analysis in 16 hospitals in Andalusia (Spain). Enferm Infecc Microbiol Clin. 2011;29(5):328-33. D0I 10.1016/j.eimc.2010.12.003.

5. Cisterna R, Ezpeleta G, Telleria 0. Nationwide sentinel surveillance of bloodstream Candida infections in 40 tertiary care hospitals in Spain. J Clin Microbiol. 2010;48(11):4200-6. DOl 10.1128/ jcm.00920-10.

6. Peman J, Canton E, Quindos G, Eraso E, Alcoba J, Guinea J, et al. Epidemiology, species distribution and in vitro antifungal susceptibility of fungaemia in a Spanish multicentre prospective survey. J Antimicrob Chemother. 2012;67(5):1181-7. DOI 10.1093/jac/ dks019.

7. Calandra T, Roberts JA, Antonelli M, Bassetti M, Vincent JL. Diagnosis and management of invasive candidiasis in the ICU: an updated approach to an old enemy. Crit Care (London, England). 2016;20(1):125. DOI 10.1186/s13054-016-1313-6.

8. Pitarch A, Nombela C, Gil C. Diagnosis of Invasive Candidiasis: From Gold Standard Methods to Promising Leading-edge Technologies. Curr Top Med Chem. 2018;18(16):1375-92. DOI 10.2174/15680266 18666181025093146.

9. Lass-Florl C. How to make a fast diagnosis in invasive aspergillosis. Med Mycol 2019;57(Supplement_2):S155-s60. 10.1093/mmy/ myy103.

10. Schauwvlieghe A, de Jonge $N$, van Dijk K, Verweij PE, Bruggemann $\mathrm{RJ}$, Biemond $\mathrm{BJ}$, et al. The diagnosis and treatment of invasive aspergillosis in Dutch haematology units facing a rapidly increasing prevalence of azole-resistance. A nationwide survey and rationale for the DB-MSG 002 study protocol. Mycoses. 2018;61(9):656-64. DOI 10.1111/myc.12788.

11. Cuenca-Estrella M. Laboratory diagnosis of fungal infection diseases. Enferm Infecc Microbiol Clin. 2012;30(5):257-64. DOI 10.1016/j. eimc.2012.01.019.

12. Clancy CJ, Nguyen MH. Diagnosing Invasive Candidiasis. J Clin Microbiol. 2018;56(5). DOI 10.1128/jcm.01909-17.

13. Patterson TF, Donnelly JP. New Concepts in Diagnostics for Invasive Mycoses: Non-Culture-Based Methodologies. J Fungi (Basel, Switzerland). 2019;5(1). DOI 10.3390/jof5010009.

14. Ayats J, Martin-Mazuelos E, Peman J, Quindos G, Sanchez F, Garcia-Rodriguez J, et al. Spanish Society of Clinical Microbiology and Infectious Diseases (SEIMC) guidelines for the diagnosis of invasive fungal infections. 2010 update. Enferm Infecc Microbiol Clin. 2011;29(1):39.e1-15. DOI 10.1016/j.eimc.2010.08.005

15. Wei $S$, Wu T, Wu Y, Ming D, Zhu X. Diagnostic accuracy of Candida albicans germ tube antibody for invasive candidiasis: sys- 
tematic review and meta-analysis. Diagn Microbiol Infect Dis. 2019;93(4):339-45. DOI 10.1016/j.diagmicrobio.2018.10.017.

16. Zaragoza $R$, Peman J, Quindos $G$, Iruretagoyena JR, Cuetara MS, Ramirez $\mathrm{P}$, et al. Kinetic patterns of Candida albicans germ tube antibody in critically ill patients: influence on mortality. Clin Vaccine Immunol. 2009;16(10):1527-8. D0I 10.1128/cvi.00183-09

17. Leon C, Ruiz-Santana S, Saavedra P, Castro C, Loza A, Zakariya I, et al. Contribution of Candida biomarkers and DNA detection for the diagnosis of invasive candidiasis in ICU patients with severe abdominal conditions. Crit care (London, England). 2016;20(1):149. DOI 10.1186/s13054-016-1324-3.

18. Munoz $P$, Vena $A$, Machado M, Gioia F, Martinez-Jimenez MC,

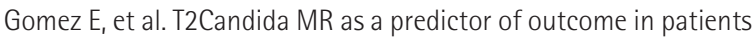
with suspected invasive candidiasis starting empirical antifungal treatment: a prospective pilot study. J Antimicrob Chemother. 2018;73(suppl_4):iv6-iv12. D0I 10.1093/jac/dky047

19. Martinez-Jimenez MC, Munoz P, Valerio M, Vena A, Guinea J, Bouza E. Combination of Candida biomarkers in patients receiving empirical antifungal therapy in a Spanish tertiary hospital: a potential role in reducing the duration of treatment. J Antimicrob Chemother. 2016;71(9):2679. DOI 10.1093/jac/dkw193.

20. Duarte RF, Sanchez-Ortega I, Cuesta I, Arnan M, Patino B, Fernandez de Sevilla $A$, et al. Serum galactomannan-based early detection of invasive aspergillosis in hematology patients receiving effective antimold prophylaxis. Clin Infect Dis. 2014;59(12):1696-702. DOI 10.1093/cid/ciu673.

21. Vena A, Bouza E, Alvarez-Uria A, Gayoso J, Martin-Rabadan P, Ca-

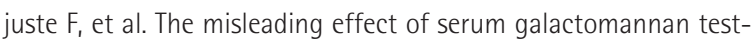
ing in high-risk haematology patients receiving prophylaxis with micafungin. Clin Microbiol Infect. 2017;23(12):1000.e1-.e4. DOI 10.1016/j.cmi.2017.05.006.

22. Duarte RF, Sanchez-Ortega I, Arnan M, Patino B, Ayats J, Sureda A, et al. Serum galactomannan surveillance may be safely withdrawn from antifungal management of hematology patients on effective antimold prophylaxis: a pilot single-center study. Bone Marrow Transplant. 2017;52(2):326-9. DOI 10.1038/bmt.2016.279.

23. Heng SC, Morrissey O, Chen SC, Thursky K, Manser RL, Nation RL, et al. Utility of bronchoalveolar lavage fluid galactomannan alone or in combination with PCR for the diagnosis of invasive aspergillosis in adult hematology patients: a systematic review and meta-analysis. Crit Rev Microbiol. 2015;41(1):124-34. DOI .3109/1040841 x.2013.804033.

24. Fortun J, Martin-Davila P, Gomez Garcia de la Pedrosa E, Silva JT, Garcia-Rodriguez J, Benito D, et al. Galactomannan in bronchoalveolar lavage fluid for diagnosis of invasive aspergillosis in non-hematological patients. J Infect. 2016;72(6):738-44. DOI 10.1016/j. jinf.2016.02.019.

25. Chong GM, Maertens JA, Lagrou K, Driessen GJ, Cornelissen JJ, Rijnders BJ. Diagnostic Performance of Galactomannan Antigen Testing in Cerebrospinal Fluid. J Clin Microbiol. 2016;54(2):428-31. D0I 10.1128/jcm.02913-15.

26. Dichtl K, Wagener J, Tschop J, Ney L. Analysis of peritoneal galactomannan for the diagnosis of Aspergillus peritonitis. Infection. 2016;44(5):683-6. DOI 10.1007/s15010-016-0902-2.
27. Kaya H, Ozaki J, Okumura H. Usefulness of Aspergillus Galactomannan Antigen Testing and the Prediction of an Outbreak during Hospital Reconstruction. Intern Med (Tokyo, Japan). 2018;57(14):19838. DOI 10.2169/internalmedicine.0269-17.

28. Zaragozá R, Peman J, Salavert M, Viudes A, Sole A, Jarque I, et al. Multidisciplinary approach to the treatment of invasive fungal infections in adult patients. Prophylaxis, empirical, preemptive or targeted therapy, which is the best in the different hosts? Ther Clin Risk Manag. 2008;4(6):1261-80. DOI: 10.2147/tcrm.s3994

29. Tran T, Beal SG. Application of the 1,3-beta-D-Glucan (Fungitell) Assay in the Diagnosis of Invasive Fungal Infections. Arch Pathol Lab Med. 2016;140(2):181-5. DOI 10.5858/arpa.2014-0230-RS.

30. Odabasi Z, Mattiuzzi G, Estey E, Kantarjian H, Saeki F, Ridge RJ, et al. Beta-D-glucan as a diagnostic adjunct for invasive fungal infections: validation, cutoff development, and performance in patients with acute myelogenous leukemia and myelodysplastic syndrome. Clin Infect Dis. 2004;39(2):199-205. DOI 10.1086/421944.

31. Tissot $F$, Lamoth $F$, Hauser $P M$, Orasch $C$, Fluckiger $U$, Siegemund $M$, et al. Beta-glucan antigenemia anticipates diagnosis of blood culture-negative intraabdominal candidiasis. Am J Resp Crit Care Med. 2013;188(9):1100-9. DOI 10.1164/rccm.201211-20690C.

32. Pickering JW, Sant HW, Bowles CA, Roberts WL, Woods GL. Evaluation of a (1->3)-beta-D-glucan assay for diagnosis of invasive fungal infections. J Clin Microbiol. 2005;43(12):5957-62. DOI 10.1128/ jcm.43.12.5957-5962.2005.

33. Albert O, Toubas D, Strady C, Cousson J, Delmas C, Vernet V, et al. Reactivity of (1-->3)-beta-d-glucan assay in bacterial bloodstream infections. Eur J Clin Microbiol Infect Dis. 2011;30(11):1453-60. DOI: 10.1007/s10096-011-1244-8

34. Ellis M, Al-Ramadi B, Finkelman M, Hedstrom U, Kristensen J, Ali-Za-

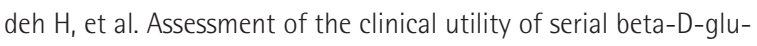
can concentrations in patients with persistent neutropenic fever. J Med Microbiol. 2008;57(Pt 3):287-95. DOI 10.1099/jmm.0.47479-0.

35. Ullmann AJ, Aguado JM, Arikan-Akdagli S, Denning DW, Groll AH, Lagrou $K_{1}$ et al. Diagnosis and management of Aspergillus diseases: executive summary of the 2017 ESCMID-ECMM-ERS guideline. Clin Microbiol Infect. 2018;24 Suppl 1:e1-e38. DOI 10.1016/j. cmi.2018.01.002.

36. Mellinghoff SC, Panse J, Alakel N, Behre G, Buchheidt D, Christopeit $M$, et al. Primary prophylaxis of invasive fungal infections in patients with haematological malignancies: 2017 update of the recommendations of the Infectious Diseases Working Party (AGIHO) of the German Society for Haematology and Medical Oncology (DGHO). Ann Hematol.2018;97(2):197-207. DOI 10.1007/s00277-017-3196-2.

37. Karageorgopoulos DE, Vouloumanou EK, Ntziora F, Michalopoulos A, Rafailidis Pl, Falagas ME. Beta-D-glucan assay for the diagnosis of invasive fungal infections: a meta-analysis. Clin Infect Dis. 2011;52(6):750-70. DOI 10.1093/cid/ciq206

38. Lamoth F, Cruciani M, Mengoli C, Castagnola E, Lortholary O, Richardson $M_{1}$ et al. Beta-Glucan antigenemia assay for the diagnosis of invasive fungal infections in patients with hematological malignancies: a systematic review and meta-analysis of cohort studies from the Third European Conference on Infections in Leu- 
kemia (ECIL-3). Clin Infect Dis. 2012;54(5):633-43. D0I 10.1093/cid/ cir897.

39. Hou TY, Wang SH, Liang SX, Jiang WX, Luo DD, Huang DH. The Screening Performance of Serum 1,3-Beta-D-Glucan in Patients with Invasive Fungal Diseases: A Meta-Analysis of Prospective Cohort Studies. PloS one. 2015;10(7):e0131602. DOI 10.1371/journal. pone.0131602.

40. Karageorgopoulos DE, Qu JM, Korbila IP, Zhu YG, Vasileiou VA, Falagas ME. Accuracy of beta-D-glucan for the diagnosis of Pneumocystis jirovecii pneumonia: a meta-analysis. Clin Microbiol Infect. 2013;19(1):39-49. DOI 10.1111/j.1469-0691.2011.03760.x.

41. Nucci $M$, Barreiros $G$, Reis $H$, Paixao M, Akiti T, Nouer SA. Performance of 1,3-beta-D-glucan in the diagnosis and monitoring of invasive fusariosis. Mycoses. 2019;62(7):570-5. D0I 10.1111/ myc.12918.

42. Shi XY, Liu Y, Gu XM, Hao SY, Wang YH, Yan D, et al. Diagnostic value of (1 --> 3)-beta-D-glucan in bronchoalveolar lavage fluid for invasive fungal disease: A meta-analysis. Resp Med. 2016;117:4853. DOI 10.1016/j.rmed.2016.05.017

43. Bhaskaran $A$, Kabbani $D$, Singer $L G$, Prochnow $T$, Bhimji A, Rotstein $C_{\text {, et al. }}(1,3)$ beta-D-Glucan in Bronchoalveolar Lavage of Lung Transplant Recipients for the Diagnosis of Invasive Pulmonary Aspergillosis. Med Mycol. 2017;55(2):173-9. DOI 10.1093/mmy/ myw052.

44. Pini P, Venturelli $C$, Girardis M, Forghieri F, Blasi E. Prognostic Potential of the Panfungal Marker (1 --> 3)-beta-D-Glucan in Invasive Mycoses Patients. Mycopathologia. 2019;184(1):147-50. D0I 10.1007/s11046-018-0282-5.

45. Martinez-Jimenez MC, Munoz $P$, Valerio $M$, Alonso $R$, Martos $C_{\text {, }}$ Guinea J, et al. Candida biomarkers in patients with candidaemia and bacteraemia. J Antimicrob Chemother. 2015;70(8):2354-61. DOI 10.1093/jac/dkv090.

46. Pini P, Colombari B, Marchi E, Castagnoli A, Venturelli C, Sarti $M$, et al. Performance of Candida albicans germ tube antibodies (CAGTA) and its association with (1-->3)-beta-D-glucan (BDG) for diagnosis of invasive candidiasis (IC). Diagn Microbiol Infect Dis.2019;93(1):39-43. DOI 10.1016/j.diagmicrobio .2018.07.007.

47. Giacobbe DR, Del Bono V, Viscoli C, Mikulska M. Use of 1,3-beta-D-glucan in invasive fungal diseases in hematology patients. Expert Rev Anti Infect Ther. 2017;15(12):1101-12. D0I 10.1080/14787210.2017.1401467.

48. Pini P, Bettua C, Orsi CF, Venturelli C, Forghieri F, Bigliardi S, et al. Evaluation of serum (1 --> 3)-beta-D-glucan clinical performance: kinetic assessment, comparison with galactomannan and evaluation of confounding factors. Infection. 2016;44(2):223-33. DOI 10.1007/s15010-015-0849-8.

49. Zhang L, Guo Z, Xie S, Zhou J, Chen G, Feng J, et al. The performance of galactomannan in combination with 1,3-beta-D-glucan or aspergillus-lateral flow device for the diagnosis of invasive aspergillosis: Evidences from 13 studies. Diagn Microbiol Infect Dis.2019;93(1):44-53. DOI 10.1016/j.diagmicrobio .2018.08.005.

50. Rouze A, Loridant S, Poissy J, Dervaux B, Sendid B, Cornu M, et al. Biomarker-based strategy for early discontinuation of empir- ical antifungal treatment in critically ill patients: a randomized controlled trial. Intensive Care Med. 2017;43(11):1668-77. D0I 10.1007/s00134-017-4932-8.

51. Barnes RA. Directed therapy for fungal infections: focus on aspergillosis. J Antimicrob Chemother. 2013;68(11):2431-4. DOI 10.1093/jac/dkt227.

52. Arvanitis M, Anagnostou T, Mylonakis E. Galactomannan and Polymerase Chain Reaction-Based Screening for Invasive Aspergillosis Among High-Risk Hematology Patients: A Diagnostic Meta-analysis. Clin Infect Dis. 2015;61(8):1263-72. DOI 10.1093/cid/civ555.

53. Martinez-Jimenez MC, Munoz P, Valerio M, Vena A, Guinea J, Bouza E. Combination of Candida biomarkers in patients receiving empirical antifungal therapy in a Spanish tertiary hospital: a potential role in reducing the duration of treatment. J Antimicrob Chemother. 2015;70(11):3107-15. DOI 10.1093/jac/dkv241.

54. Posteraro $B$, Tumbarello M, De Pascale $G$, Liberto E, Vallecoccia MS, De Carolis $E_{1}$ et al. $(1,3)$-beta-d-Glucan-based antifungal treatment in critically ill adults at high risk of candidaemia: an observational study. J Antimicrob Chemother. 2016;71(8):2262-9. D0I 10.1093/ jac/dkw112.

55. Dib RW, Hachem R, Chaftari AM, Raad I. Appropriate Duration of Intravenous Treatment of Candidemia and Timing of Step Down to Oral Therapy in Non-neutropenic Patients. Mediterr J Hematol Infect Dis. 2018;10(1):e2018028. DOI 10.4084/mjhid. 2018.028.

56. Falces-Romero I, Cendejas-Bueno E, Laplaza-Gonzalez M, Escosa-Garcia L, Schuffelmann-Gutierrez C, Calderon-Llopis B, et al. T2Candida((R)) to guide antifungal and lengh of treatment of candidemia in a pediatric multivisceral transplant recipient. Med Mycol Case Rep. 2018;21:66-8. D0I 10.1016/j.mmcr.2018.05.006.

57. Bassetti M, Garnacho-Montero J, Calandra T, Kullberg B, Dimopoulos $G$, Azoulay $E$, et al. Intensive care medicine research agenda on invasive fungal infection in critically ill patients. Intensive Care Med. 2017;43(9):1225-38. DOI 10.1007/s00134-017-4731-2.

58. Bassetti M, Giacobbe DR, Vena A, Trucchi C, Ansaldi F, Antonelli M, et al. Incidence and outcome of invasive candidiasis in intensive care units (ICUs) in Europe: results of the EUCANDICU project. Crit Care (London, England). 2019;23(1):219. D0I 10.1186/s13054-0192497-3.

59. Martin-Loeches I, Antonelli M, Cuenca-Estrella M, Dimopoulos G, Einav S, De Waele JJ, et al. ESICM/ESCMID task force on practical management of invasive candidiasis in critically ill patients. Intensive Care Med. 2019;45(6):789-805. DOI 10.1007/s00134-01905599-w.

60. Hage CA, Carmona EA, Epelbaum O, Evans SE, Gabe LM, Haydour O, Knox KS, et al. Microbiological laboratory testing in the diagnosis of fungal infections in pulmonary and critical care practice. An official American Thoracic Society Clinical Practice Guideline. Ann Am Thorac Soc. 2019 Sep 17.DOI 10.1513/AnnalsATS.201908-582CME. 